\title{
BMJ Open Randomised controlled trial of rhinothermy for treatment of the common cold: a feasibility study
}

\author{
Susanne van de Hei, ${ }^{1,2}$ Steven McKinstry, ${ }^{1,3,4}$ George Bardsley, ${ }^{1,4}$ \\ Mark Weatherall, ${ }^{4,5}$ Richard Beasley, ${ }^{1,3,4}$ James Fingleton ${ }^{1,3,4}$
}

To cite: Hei Sv, McKinstry S, Bardsley G, et al.

Randomised controlled trial of rhinothermy for treatment of the common cold: a feasibility study. BMJ Open 2018;8:e019350. doi:10.1136/ bmjopen-2017-019350

- Prepublication history and additional material for this paper are available online. To view these files, please visit the journal online (http://dx.doi. org/10.1136/bmjopen-2017019350).

Received 28 August 2017 Revised 21 February 2018 Accepted 28 February 2018

Check for updates

${ }^{1}$ Medical Research Institute of New Zealand, Wellington, New Zealand

${ }^{2}$ University of Groningen, Groningen, The Netherlands

${ }^{3}$ Victoria University of Wellington, Wellington, New Zealand

${ }^{4}$ Capital and Coast District Health Board, Wellington, New

Zealand

${ }^{5}$ University of Otago, Wellington, New Zealand

Correspondence to

Dr James Fingleton;

james.fingleton@mrinz.ac.nz

\section{ABSTRACT}

Objective To determine the feasibility of a randomised controlled trial (RCT) of rhinothermy for the common cold. Design Open label, randomised, controlled feasibility study.

Setting Single-centre research institute in New Zealand recruiting participants from the community.

Participants 30 adult participants with symptoms of a common cold, presenting within 48 hours of the onset of symptoms.

Interventions Participants were randomly assigned 2:1 to receive either $35 \mathrm{~L} / \mathrm{min}$ of $100 \%$ humidified air at $41^{\circ} \mathrm{C}$ via high flow nasal cannulae, 2 hours per day for up to 5 days (rhinothermy), or vitamin C $250 \mathrm{mg}$ daily for 5 days (control).

Primary and secondary outcome measures The primary outcome was the proportion of screened candidates who were randomised. Secondary outcomes included: proportion of randomised participants who completed the study; modified Jackson scores from randomisation to 10 days after initiation of randomised regimen; time until feeling 'a lot better' compared with study entry; time until resolution of symptoms or symptom score at 10 days postrandomisation; proportion of organisms identified by PCR analysis of nasal swabs taken at baseline; the patterns of use of the rhinothermy device; estimated adherence of the control group; and rhinothermy device tolerability.

Results In all $30 / 79$ (38\%, $95 \% \mathrm{Cl} 27 \%$ to $50 \%$ ) of potential participants screened for eligibility were randomised. Rhinothermy was well tolerated, and all randomised participants completed the study $(100 \%$, $95 \% \mathrm{Cl} 88 \%$ to $100 \%$ ). The reduction from baseline in the modified Jackson score was greater with rhinothermy compared with control at days 2, 3, 4, 5 and 6 , with the maximum difference at day $4(-6.4,95 \% \mathrm{Cl}-9.4$ to -3.3$)$. The substantial clinical benefit threshold for modified Jackson score was a 5-unit change.

Conclusions This study shows that an RCT of rhinothermy compared with low-dose vitamin $\mathrm{C}$ in the treatment of the common cold is feasible.

Trial registration number ACTRN12616000470493; Results.

\section{INTRODUCTION}

The common cold is the most frequent infection worldwide. Adults report between two

\section{Strengths and limitations of this study}

This is the first study investigating the use of rhinothermy delivered via high flow nasal cannulae for the treatment of the common cold.

- This open-label, randomised controlled feasibility study shows that a randomised controlled trial of rhinothermy compared with low-dose vitamin $\mathrm{C}$ in the treatment of the common cold is feasible.

- A5-unit change in modified Jackson score has been estimated to represent the threshold for substantial clinical benefit.

- Although this study suggested a benefit from rhinothermy, this finding should be considered with caution, as this was a feasibility study in which efficacy was not the primary outcome measure.

- A robust adequately powered RCT is needed, particularly as this feasibility study identified that rhinothermy improved symptoms of the common cold compared with control.

and four colds a year and children between six and eight colds a year. ${ }^{1}$ The median incubation period depends on the virus but is usually between 1 and 6 days. $^{2}$ The severity of symptoms typically peaks 3 to 3 days after symptom onset. ${ }^{1}$ It is almost always a self-limiting illness, and the total symptom duration is between 7 and 14 days. ${ }^{3}$ Historical estimates put the total cost of the common cold in the USA as high as $\$ 40$ billion per year. ${ }^{4}$ This includes both healthcare resource use and work productivity losses, the latter approaching $\$ 25$ billion. ${ }^{5}$ About one half of common colds are caused by human rhinovirus (HRV), ${ }^{36}$ although in autumn, HRV may account for $80 \%$ of all upper respiratory tract infections. ${ }^{6} \mathrm{HRV}$ is strongly associated with exacerbations of asthma in both children and adults and causes more severe and long-lasting symptoms in these patient groups. $^{7}$ Respiratory viruses, including HRV, are found in $40 \%$ of exacerbations in patients with chronic obstructive pulmonary disease (COPD). ${ }^{8}$ 
Treatment for the common cold is supportive and symptomatic. About two-thirds of those with a cold use 'over the counter' products. ${ }^{4}$ Treatments that have not shown benefit in the treatment of the common cold include: echinacea, antihistamines, intranasal corticosteroids, antivirals and antibiotics. ${ }^{39-12}$ Regular supplementation with vitamin $\mathrm{C}$ has a small effect on cold duration, but therapeutic supplementation once symptoms develop lacks evidence of efficacy, ${ }^{13}$ and it is unlikely that supplementation with low-dose vitamin $\mathrm{C}$ once symptomatic will reduce symptom severity or duration. There is no effective HRV vaccine, in part because of the high sequence variability in the antigenic sites of the 150 distinct HRV serotypes. $^{14}$

Steam inhalation for symptom relief is a traditional remedy for the common cold. There is a scientific rationale for this treatment because HRV is a temperature-sensitive virus with temperature-dependent reduction in viral replication between $33^{\circ} \mathrm{C}$ and $43^{\circ} \mathrm{C}$ in vitro. ${ }^{15-18}$ Host defence may also be enhanced at higher temperatures through mechanisms, such as greater production and responsiveness to interferon, ${ }^{19}{ }^{20}$ and enhanced antiviral immune responses mediated by heat shock proteins. ${ }^{15}$ Clinical studies of the efficacy of heated humidified air delivered at high flow to the upper airways (rhinothermy) report inconsistent results. ${ }^{16}{ }^{21-25}$ Study limitations include variable, and likely ineffective, methods of delivering heated humid air at high flow to the upper airways. These methods include delivery through nozzles held a distance away from the nares and vented anaesthetic masks. The reported intervention durations were also variable. A Cochrane Review assessed that the current evidence could not support or refute effectiveness and recommended that there is a need for robustly designed and adequately powered randomised controlled trials (RCTs) to assess the effectiveness of rhinothermy to treat the common cold. ${ }^{21}$

We have reported a tolerability study that found that a high flow nasal cannula device (myAIRVO 2, Fisher \& Paykel Healthcare, Auckland, New Zealand) modified to deliver high flow humidified air at $41^{\circ} \mathrm{C}$ via wide bore nasal cannulae was well tolerated in healthy volunteers. ${ }^{26}$ The potential advantage of this device for rhinothermy is that it can deliver a consistent temperature, humidity and flow rate direct into the upper airways. The study reported here is a feasibility study of rhinothermy in the treatment of the common cold that will inform the design of a robust RCT of this treatment. The specific aims of the feasibility study were to:

1. estimate the proportions of potential participants after screening who undergo randomisation, and who then complete the study, in turn providing an estimate of the time and resources required to undertake an RCT;

2. estimate the $\mathrm{SD}$ of the proposed primary outcome variable for the RCT, the modified Jackson score, ${ }^{27-29}$ so that the sample size for the RCT can be better estimated;
3. estimate the likely minimum clinically important difference (MCID) in modified Jackson score among people with the common cold who are eligible to take part in the study;

4. estimate the proportions of the different viruses causing the cold among people with the common cold who are eligible to take part in the study;

5. examine the use and tolerability of the modified myAIRVO 2 device in those with the common cold.

\section{METHODS \\ Study design}

An open-label, randomised, controlled feasibility study.

\section{Participants}

Participants with self-reported symptoms of the common cold were recruited from the community by advertisement on Wellington Hospital (New Zealand) notice boards and intranet, local primary care practices, pharmacies, student notice boards, social media and from the Medical Research Institute of New Zealand database.

Participants were eligible if they were between 16 and 75 years old, had common cold symptoms for less than 48 hours at the time of randomisation and a modified Jackson score $\geq 5 .{ }^{28-30}$ The modified Jackson score is calculated from a symptom severity questionnaire that consists of eight symptoms: sneezing, nasal discharge, nasal congestion, sore/scratchy throat, cough, headache, malaise and fever/chills, which are scored on a scale from 0 to $3(0=$ absent, $1=$ mild, $2=$ moderate, $3=$ severe $)$. The total score can range from 0 to 24 .

Participants were not recruited if they had: (1) an immunosuppressive medical condition and/or were prescribed immunosuppressive medications; (2) a diagnosis of asthma, COPD or other significant respiratory conditions; (3) nasal conditions such as a deviated septum or chronic rhinitis, which could impair nasal breathing; (4) use of cold remedies, for example, decongestants/ cough medicines, linctus/throat lozenges, within 6 hours of randomisation; (5) currently using antibiotics or inhaled medication; (6) known allergy to ascorbic acid (vitamin C); (7) the investigator believed the participant or their caregiver would be unable to safely use the myAIRVO 2 device without medical supervision; (8) had any other condition which, at the investigator's discretion, was believed may present a safety risk or impact the feasibility of the study or the study results.

The study was prospectively registered on the Australian New Zealand Clinical Trials Registry (Trial ID: ACTRN12616000470493). The study was sponsored by Fisher \& Paykel Healthcare, the manufacturer of the myAIRVO 2 device. Written informed consent was obtained before starting any study specific procedures. During the study, participants were able to stop the intervention at any time, without being withdrawn from the study. Participants were also able to withdraw from the study at any time without giving a reason. 


\section{Randomisation and blinding}

Participants were randomly assigned in a 2:1 ratio to two groups that received either: (1) rhinothermy via the modified myAIRVO 2 device, 2 hours per day for up to 5 days or (2) control medication, vitamin C $250 \mathrm{mg}$ (Healtheries, Vitaco Health NZ, Auckland, New Zealand) daily for 5 days. The randomisation schedule was computer generated by the study statistician, who was not involved in study recruitment, procedures or data collection. Randomisation was administered by presealed opaque envelopes that were opened at the point of randomisation by the investigator. Investigators were blinded to allocation until this point. Due to the nature of the interventions and the unequal randomisation design, participants, investigators and the study statistician could not be masked to treatment allocation.

\section{Study procedures}

After demographic data were collected, baseline common cold symptoms were recorded using the modified Jackson score, and a physical examination was carried out by a doctor. Once eligibility was confirmed, pernasal flocked swabs (Copan Diagnostics, California, USA) were collected into Universal Transport Medium (Roche Diagnostics, Basel, Switzerland). After amplification, samples underwent nucleic acid detection using multiplex reverse transcriptase-PCR (Roche Diagnostics, Basel, Switzerland) in conjunction with the Respiratory Pathogens 21 kit (Fast-track Diagnostics, Junglinster, Luxembourg). This allowed the detection, if present, of influenza A, influenza A (H1N1), influenza B, rhinovirus, coronavirus NL63, 229E, OC43, HKU1, parainfluenza 1, 2, 3, 4, human metapneumovirus A/B, human bocavirus, respiratory syncytial virus $\mathrm{A} / \mathrm{B}$, adenovirus, enterovirus, parechovirus and mycoplasma pneumoniae.

The intervention group received rhinothermy via the modified myAIRVO 2 device (PT100AZ, Fisher \& Paykel Healthcare), a heated breathing tube and chamber (900PT551, Fisher \& Paykel Healthcare), and Optiflow+nasalcannula (OPT944, Fisher \& Paykel Healthcare; see figure 1). This system is a humidifier with an integrated flow generator, able to humidify respiratory gases and deliver them down a heated breathing tube through the nasal cannula interface. After randomisation, participants allocated to the intervention group used the myAIRVO 2, inhaling $100 \%$ humidified air, at $41^{\circ} \mathrm{C}$, at $35 \mathrm{~L} /$ min continuously for 2 hours, under supervision of investigators at the Clinical Trials Unit (CTU), Wellington Hospital (Wellington, New Zealand).

After training on myAIRVO 2 setup and use, participants were given a modified myAIRVO 2 device to use at home according to the same regimen ( $100 \%$ humidity, $41^{\circ} \mathrm{C}, 35 \mathrm{~L} / \mathrm{min}, 120 \mathrm{~min}$ ) for up to 5 days depending on resolution of symptoms. Participants were encouraged to use the device for at least 2 hours per day and longer if desired in either a single or repeated administration, preferably in the evening. Participants were allowed to reduce the flow to $30 \mathrm{~L} / \mathrm{min}$ or $25 \mathrm{~L} / \mathrm{min}$ according to comfort when at home. After 3 days, participants that were randomised to the myAIRVO 2 group were contacted by phone to check if they had any adverse events and/or had any problems with using the myAIRVO 2.

Participants allocated to the control group received their first $250 \mathrm{mg}$ vitamin $\mathrm{C}$ tablet in the CTU after randomisation, and then at home for a further 4 days. Low-dose vitamin $\mathrm{C}$ was used as a control. As therapeutic use of vitamin $\mathrm{C}$ after symptoms develop has not been shown to consistently reduce the duration of colds or improve cold symptoms, ${ }^{14}$ participants were not expected to derive benefit above that obtained by the placebo effect. The low dose of vitamin $\mathrm{C}$ used in this study is freely available in pharmacies in New Zealand but is unlikely to be of benefit when taken after symptoms develop, as opposed to regular supplementation. Participants who used vitamin $\mathrm{C}$ returned the bottle for medicines reconciliation.

All participants were requested to complete a symptom diary every day for 10 days. The diary comprised a daily

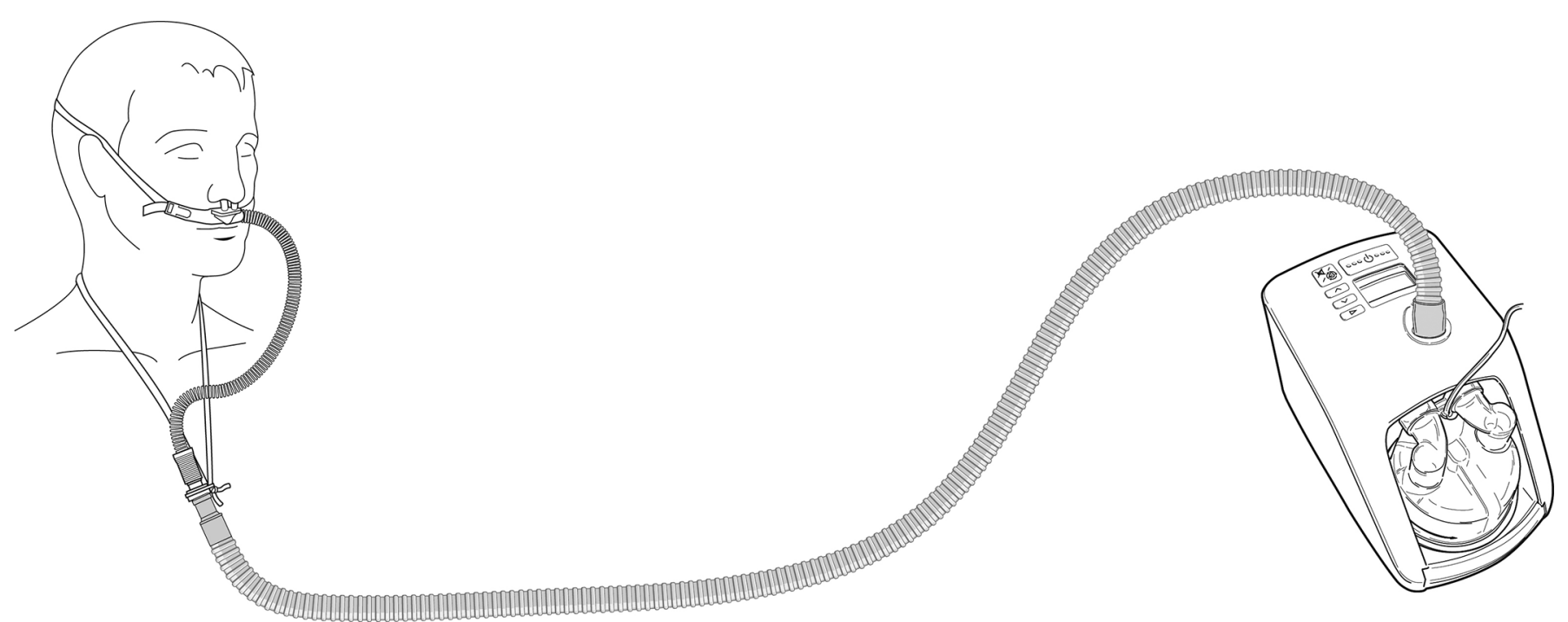

Figure 1 Illustration of 'myAIRVO 2 and Optiflow+cannula' setup. 
modified Jackson score questionnaire and a box to tick when they first felt 'a lot better' compared with study entry. The mean change in modified Jackson score when participants felt 'a lot better' was used to estimate the MCID.

All participants returned to the clinic for a second and final visit after day 10 and returned their symptom diary. Participants who used myAIRVO 2 returned the device and were asked to fill in a tolerability questionnaire about their experience. There were nine questions, with participants recording their answer on a continuous scale from most positive (0) to least positive (100). The questions were about: (1) the teaching session; (2) connecting the components; (3) cleaning process; (4) level of comfort; (5) amount of moisture from the device; (6) weight of the nasal interface; (7) noisiness; (8) applying and removing the nasal interface; and (9) the likelihood of reusing it in the future. Compliance data from the myAIRVO 2 were uploaded and analysed.

\section{Outcomes}

The primary outcome measure was the proportion of recruits who were randomised. Other outcome measures included: (1) proportion of randomised participants who completed the study; (2) mean and SD of the modified Jackson scores from randomisation to 10 days after initiation of the randomised regimen; (3) time until feeling 'a lot better' compared with study entry; (4) time until resolution of symptoms or symptom score at 10 days postrandomisation; (5) proportion of organisms identified by PCR analysis of nasal swabs taken at baseline; (6) the patterns of use of the myAIRVO 2 device, as determined by electronic monitoring capabilities of the myAIRVO 2; (7) estimated adherence of the control group; and (8) myAIRVO 2 tolerability questionnaire results.

\section{Sample size and statistical analysis}

A sample size of 30 was chosen to:

1. assess the proportion of screened participants who were randomised into the trial. If a minimum of 60 patients were approached, assuming a recruitment rate of $50 \%$ or less, an achieved participation of 30 participants has $80 \%$ power, one sided alpha of $5 \%$ to rule out a recruitment rate of less than $34 \%$;

2. estimate the $\mathrm{SD}$ of the change from baseline modified Jackson score. A sample size of at least 25 has acceptable precision to estimate a SD based on a $\chi^{2}$ distribution;

3. estimate the minimum clinically important difference in Jackson score. This would require a minimum of 25 participants for precision (based on a t statistic).

The recruitment and completion rates CIs were estimated by the Clopper-Pearson exact method. Mixed linear models with an exponential-in-time covariance matrix were used to estimate the differences in modified Jackson scores by treatment and time. A post hoc interaction term tested if the response in modified Jackson score differed by whether the nasal swab results was positive or not. The CI for the SD for the change from baseline modified Jackson score was estimated using a $\chi^{2}$ distribution. Logistic regression was used to estimate the receiver operating characteristic curves for the probability of participants first saying they were a lot better compared with the change in baseline modified Jackson score at that particular time. The times of measurement were chosen so that about half of participants reported they had improved by that time point. Individual sensitivities and specificities were calculated for change from baseline modified Jackson score of between -7 and -4 at these times.

Survival analysis was used to estimate the relationship between treatment and time until the participants said they felt better and time until the modified Jackson score was zero with Kaplan-Meier plots of survival and Cox proportional hazards regression to estimate the difference in survival. Note that a shorter survival is better in this analysis as the event is time to feeling better.

SAS V.9.4 was used. Study data were collected and managed using REDCap electronic data capture tools hosted at the Medical Research Institute of New Zealand. ${ }^{30}$

\section{Patient and public involvement}

No patients or public were involved in setting out the research question or developing the outcome measures, nor were they involved in developing plans for design or implementation of the study. No patients or public were asked to advise on interpretation or writing up of results, nor was the burden of the interventions on patients assessed. The results of the research will be disseminated to those study participants who wished to be notified of them.

\section{RESULTS}

\section{Participant characteristics}

Participant flow through the study is shown in figure 2. In all, 79 potential participants were screened for eligibility and 30 participants were randomised. All participants completed their visits between 12 May 2016 and 13 July 2016. Participants were predominantly young adults who were non-smokers (table 1).

\section{Recruitment rate}

The recruitment rate was $30 / 79(38.0 \%)$ with a $95 \%$ CI $27.3 \%$ to $49.6 \%$. One of the main reasons for exclusion was that participants had symptoms for 48 hours or longer at presentation (figure 2). All 30 randomised participants completed the study, resulting in a retention rate of $100 \%$ with a lower $95 \%$ confidence limit of $88.4 \%$.

\section{Symptoms}

Participants had symptoms for a mean of 31 hours at presentation, at which time the mean modified Jackson score was 11.7 (table 1). There were 27 participants who completed the modified Jackson score after 


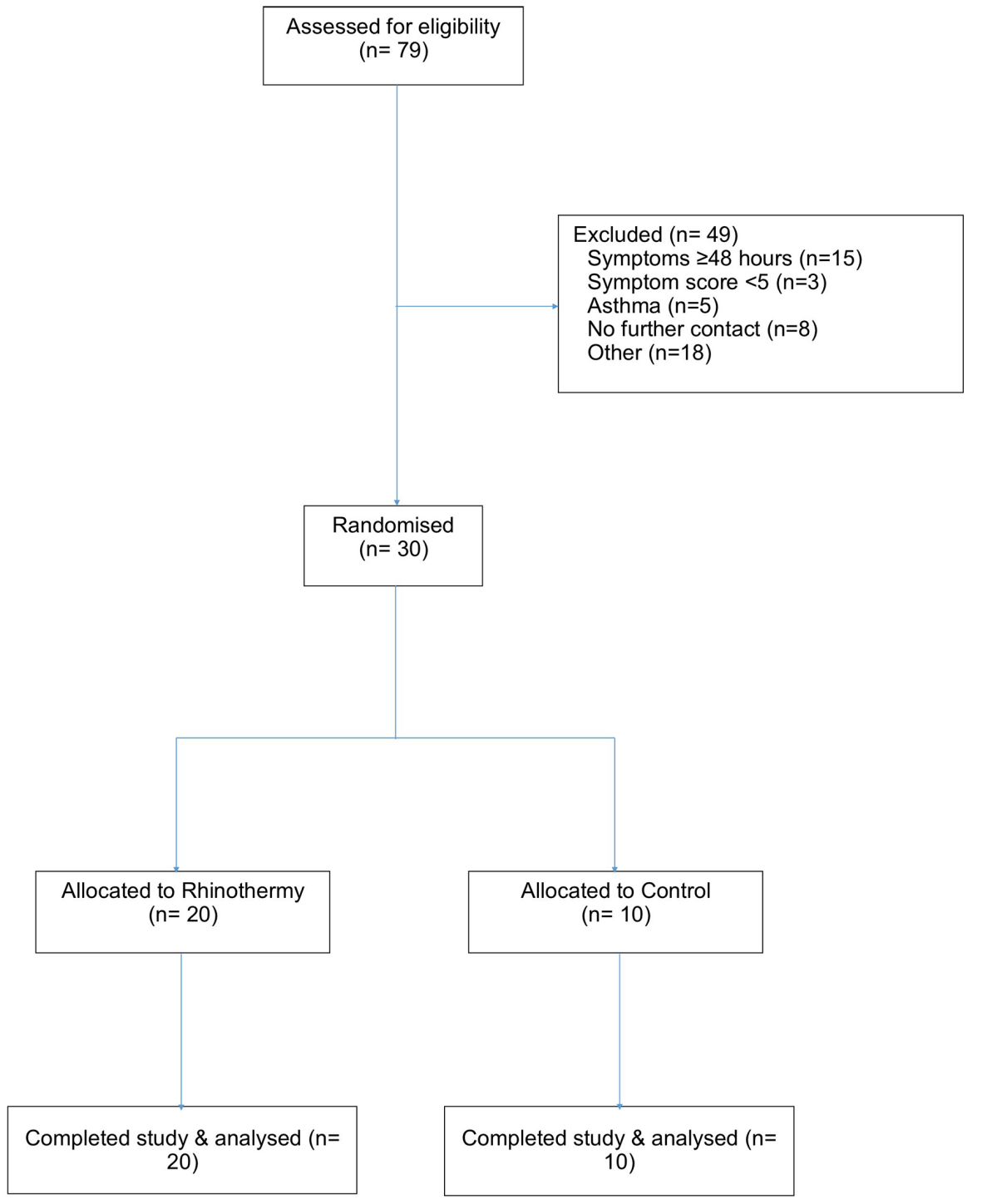

Figure 2 Participant flow through the study and allocation of intervention.

10 days. Table 2 shows the mean (SD), median (IQR) and the range of the modified Jackson score from baseline to day 10 for both groups. The mean change in modified Jackson score from baseline was greater in the rhinothermy group than in the control group after 24 hours and remained greater than the control group until day 6 (table 3 and online supplementary table S1). Figure 3 shows a plot of the change from baseline of the modified Jackson score.

\section{Estimation of MCID/substantial clinical benefit}

In total, 27 participants reported feeling 'a lot better' within 10 days of randomisation; 17/20 participants from the rhinothermy group and all 10 control group participants. Two participants did not fill in the modified Jackson score on the day they felt 'a lot better'. Therefore, an estimate of the MCID used the scores of 25 participants: 16 in the rhinothermy group and 9 in the control group. We recognised post hoc that the threshold 'a lot better' corresponded to an estimate of substantial clinical benefit rather than the MCID, ${ }^{31} 32$ and so we have estimated the change that represents substantial clinical benefit. The change from baseline modified Jackson score which had the best sensitivity and specificity for the participants stating that they felt a lot better was between 5 and 6 units on the modified Jackson Score on days 4 and 5 (table 4). Based on this analysis, we propose that a 5 -unit change in modified Jackson score is the substantial clinical benefit threshold for this instrument.

The difference in modified Jackson score between the rhinothermy group and the control group was greater than the substantial clinical benefit threshold on days 3, 4 and $5, \mathrm{P}<0.001$ (table 3).

Complete resolution of symptoms, denoted by a modified Jackson score of zero, occurred in only 12 participants within 10 days after randomisation: 10 of 19 participants who completed the modified Jackson score on day 10 in the rhinothermy group and 2 of 10 in the vitamin C group. The HR (95\% CI) for feeling 'a lot better' rhinothermy compared with control was 
Open Access

Table 1 Baseline participant characteristics

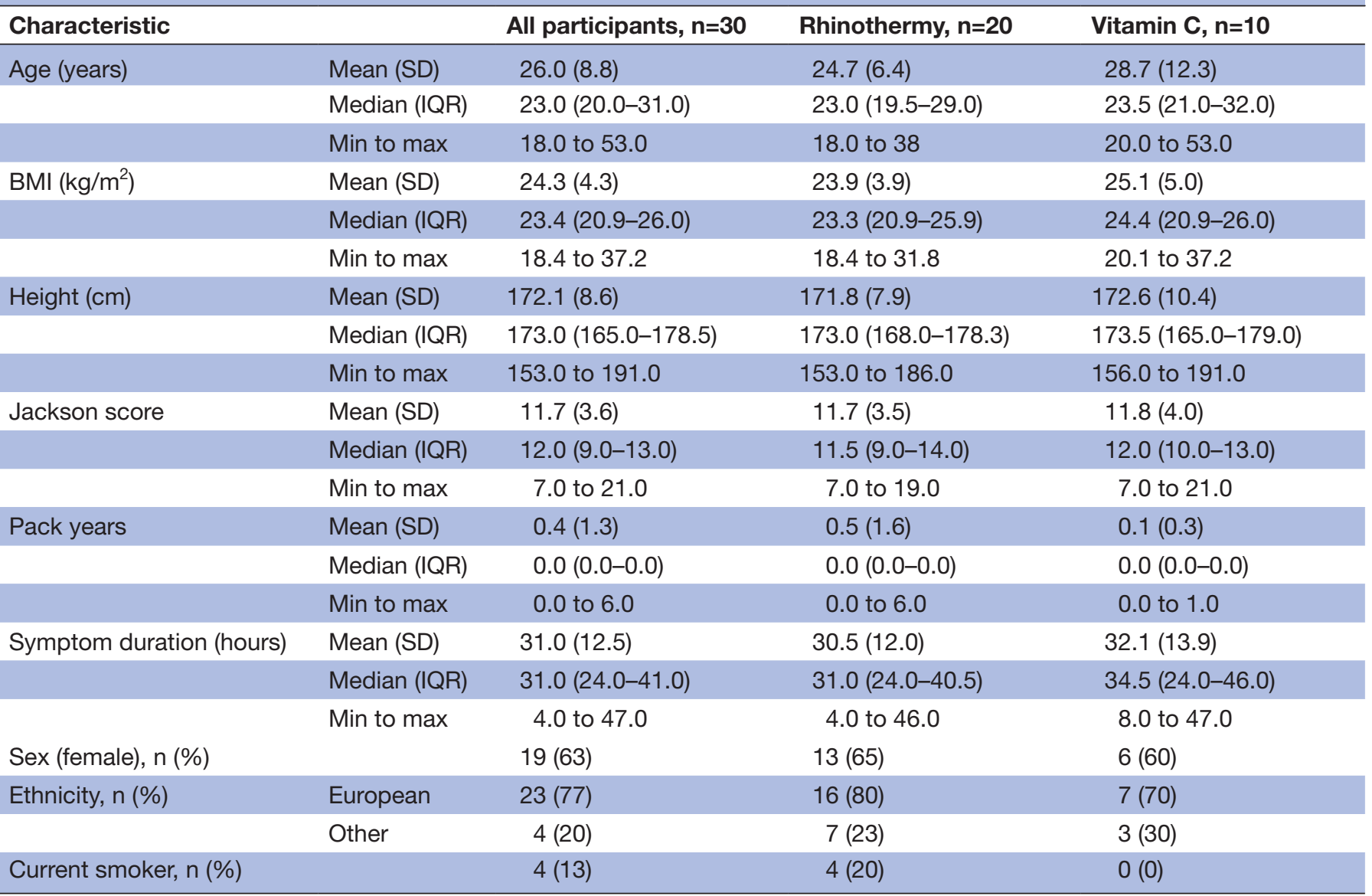

BMI, body mass index.

1.33 (0.60 to 2.98 ), $\mathrm{P}=0.48$, which favoured the rhinothermy group. Kaplan-Meier based survival curves are shown in figure 4 . There were no reported adverse events.

\section{Estimation of the SD for modified Jackson score}

The SDs for the modified Jackson score at days 4 and 5, based on the pooled values from t-tests, were $3.8(95 \%$
CI 3.0 to 5.2) and 4.8 (3.8 to 6.6) and for change from baseline modified Jackson score 3.7 (95\% CI 2.9 to 5.0) and 4.7 (3.7 to 6.5$)$.

\section{Nasal swab viral PCR results}

Positive nasal swab PCR for a respiratory virus was found in 16 participants $(53.3 \%)$. The organisms found are shown in table 5. HRV was the most commonly detected virus

Table 2 Modified Jackson score from baseline to day 10

\begin{tabular}{|c|c|c|c|c|c|c|}
\hline \multirow{2}{*}{$\begin{array}{l}\text { N } \\
\text { Rhinothermy/ } \\
\text { control }\end{array}$} & \multicolumn{3}{|c|}{ Rhinothermy } & \multicolumn{3}{|l|}{ Control } \\
\hline & Mean (SD) & Median (IQR) & Min to $\max$ & Mean (SD) & Median (IQR) & Min to $\max$ \\
\hline Baseline $n=20 / 10$ & $11.7(3.5)$ & $11.5(9-14)$ & 7 to 19 & $11.8(4.0)$ & $12(10-13)$ & 7 to 21 \\
\hline Day 2 n=19/10 & $9.4(3.9)$ & $10(7-12)$ & 2 to 16 & $13.5(3.7)$ & $12.5(11-15)$ & 10 to 22 \\
\hline Day $3 n=19 / 10$ & $7.2(3.7)$ & $7(4-10)$ & 1 to 14 & $12.3(4.0)$ & $11.5(9-15)$ & 8 to 19 \\
\hline Day $4 \mathrm{n}=19 / 10$ & $4.4(3.3)$ & $4(2-7)$ & 0 to 10 & $10.7(4.6)$ & $9(8-15)$ & 5 to 18 \\
\hline Day $6 n=18 / 10$ & $2.8(3.6)$ & $2(0-5)$ & 0 to 13 & $7.5(4.5)$ & $9.5(3-10)$ & 1 to 13 \\
\hline Day $7 n=18 / 10$ & $2.4(2.5)$ & $2(0-5)$ & 0 to 8 & $4.8(2.9)$ & $4(3-8)$ & 1 to 9 \\
\hline Day 8 n=18/9 & $1.9(2.7)$ & $0.5(0-3)$ & 0 to 10 & $3(1.7)$ & $3(2-4)$ & 0 to 5 \\
\hline Day $9 n=19 / 10$ & $2.1(2.9)$ & $1(0-3)$ & 0 to 11 & $2.4(2.1)$ & $1.5(1-3)$ & 0 to 8 \\
\hline
\end{tabular}


Table 3 Mixed linear model estimates of differences between treatments at each time point with baseline modified Jackson score as a continuous covariate

\begin{tabular}{|c|c|c|}
\hline $\begin{array}{l}\text { Day of } \\
\text { measurement }\end{array}$ & $\begin{array}{l}\text { Modified Jackson score } \\
\text { Rhinothermy minus control } \\
(95 \% \mathrm{Cl})\end{array}$ & P values \\
\hline Two & $-4.11(-6.68$ to -1.56$)$ & 0.002 \\
\hline Three & $-5.18(-7.74$ to -2.62$)$ & $<0.001$ \\
\hline Four & $-6.37(-8.93$ to -2.71$)$ & $<0.001$ \\
\hline Five & $-5.29(-7.86$ to -2.67$)$ & $<0.001$ \\
\hline Six & $-4.61(-7.19$ to -2.04$)$ & $<0.001$ \\
\hline Seven & $-2.47(-5.05$ to 0.11$)$ & 0.060 \\
\hline Eight & $-1.10(-3.73$ to 1.54$)$ & 0.41 \\
\hline Nine & $-0.28(-2.84$ to 2.28$)$ & 0.83 \\
\hline Ten & $0.14(-2.42$ to 2.70$)$ & 0.91 \\
\hline
\end{tabular}

and was found in one-third of participants. There was no evidence of an interaction between the treatment difference in modified Jackson scores for those that were nasal swab positive or negative ( $\mathrm{P} \geq 0.17$ for all time points).

\section{Patterns of rhinothermy and vitamin $\mathrm{C}$ use}

The mean total use of the myAIRVO 2 device was 9.9 hours, a mean (SD) of $2.0(0.85)$ hours per day. Eight participants (40\%) did not use the myAIRVO 2 device on day 5 and seven participants (35\%) used it for less than 4 days. On the days the myAIRVO 2 was used, the mean (SD) use was 2.6 (0.5) hours per day. All participants in the control group took all five Vitamin C tablets as per protocol ( $100 \%$ adherence).
Table 4 Sensitivity and specificity for 'feeling better' by selected change from baseline modified Jackson scores for day 4 and day 5

\begin{tabular}{cll}
\hline & \multicolumn{2}{l}{ Improved by participant report } \\
\cline { 2 - 3 } Change from baseline & Sensitivity (\%) & Specificity (\%) \\
\hline Day 4 & & \\
-7 & $9 / 11(82)$ & $15 / 18(83)$ \\
-6 & $9 / 11(82)$ & $13 / 18(72)$ \\
-5 & $11 / 11(100)$ & $12 / 18(67)$ \\
-4 & $11 / 11(100)$ & $8 / 18(44)$ \\
Day 5 & & \\
\hline-7 & $10 / 17(59)$ & $10 / 12(83)$ \\
-6 & $12 / 17(71)$ & $10 / 12(83)$ \\
-5 & $15 / 17(88)$ & $10 / 12(83)$ \\
-4 & $16 / 17(94)$ & $7 / 12(58)$ \\
\hline
\end{tabular}

On day 1 at the clinic, all participants used a flow of $35 \mathrm{~L} / \mathrm{min}$. On day 2, 13 participants kept using this flow rate at home, while 7 participants used a reduced flow; a flow of $30 \mathrm{~L} / \mathrm{min}$ and $25 \mathrm{~L} / \mathrm{min}$ was used by 3 and 4 participants, respectively. On days 3, 4 and 5, a mixture of the three different flow rates were used with a mean flow rate overall of around $32 \mathrm{~L} / \mathrm{min}$.

\section{Rhinothermy tolerability questionnaire}

Tolerability questionnaire results for the myAIRVO 2 are shown in table 6 . The device was reasonably simple to connect at home, comfortable to wear and acceptable with regards to the amount of moisture build-up

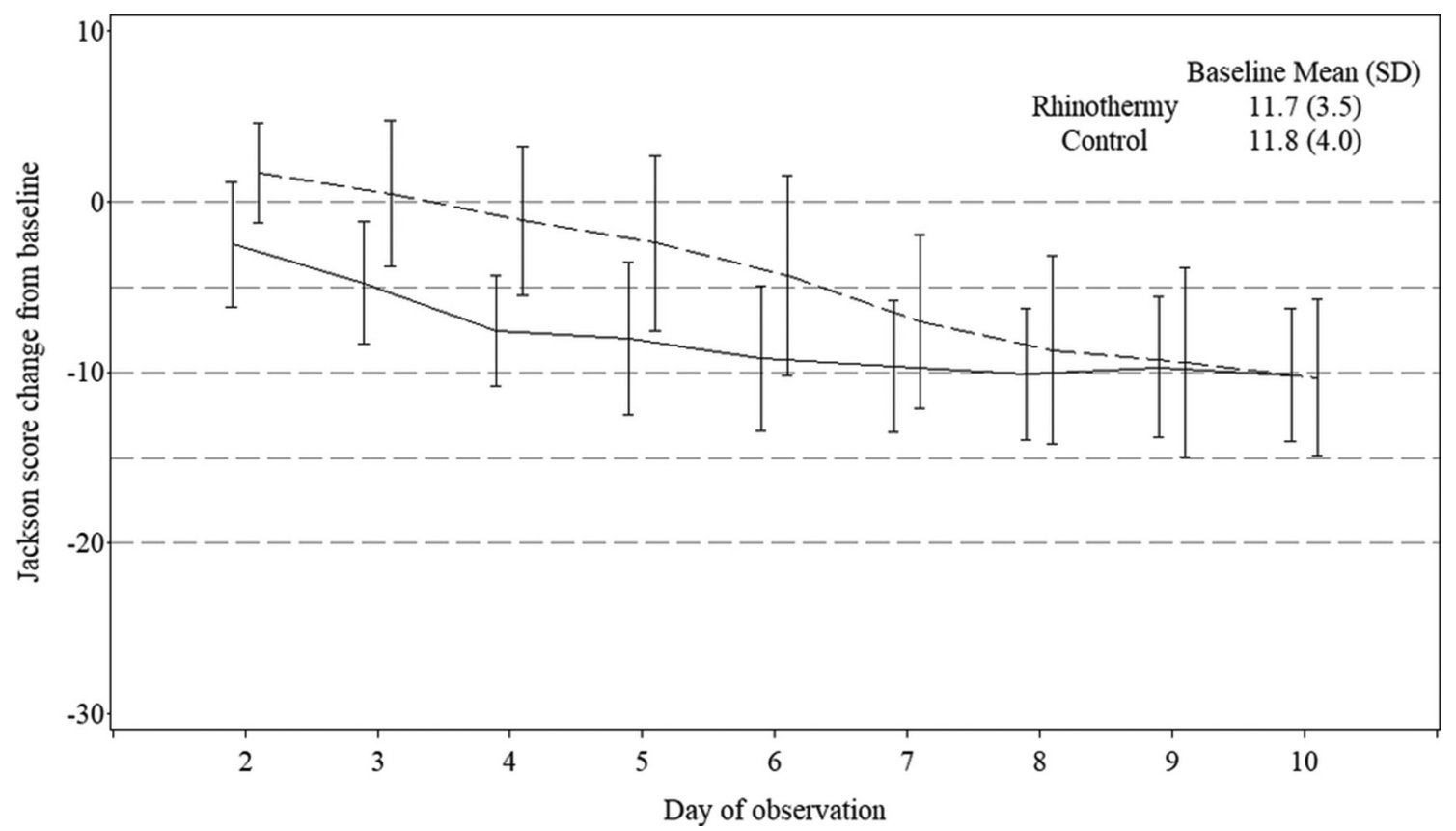

Figure 3 Plot of mean (SD) modified Jackson score change from baseline by time and treatment. Solid line represents rhinothermy group, while dotted line represents control. Y-axis shows mean modified Jackson score change from baseline. Error bars represent one SD from the mean. Mean Jackson score was the same at baseline and day 1 so data are shown from day 2. 


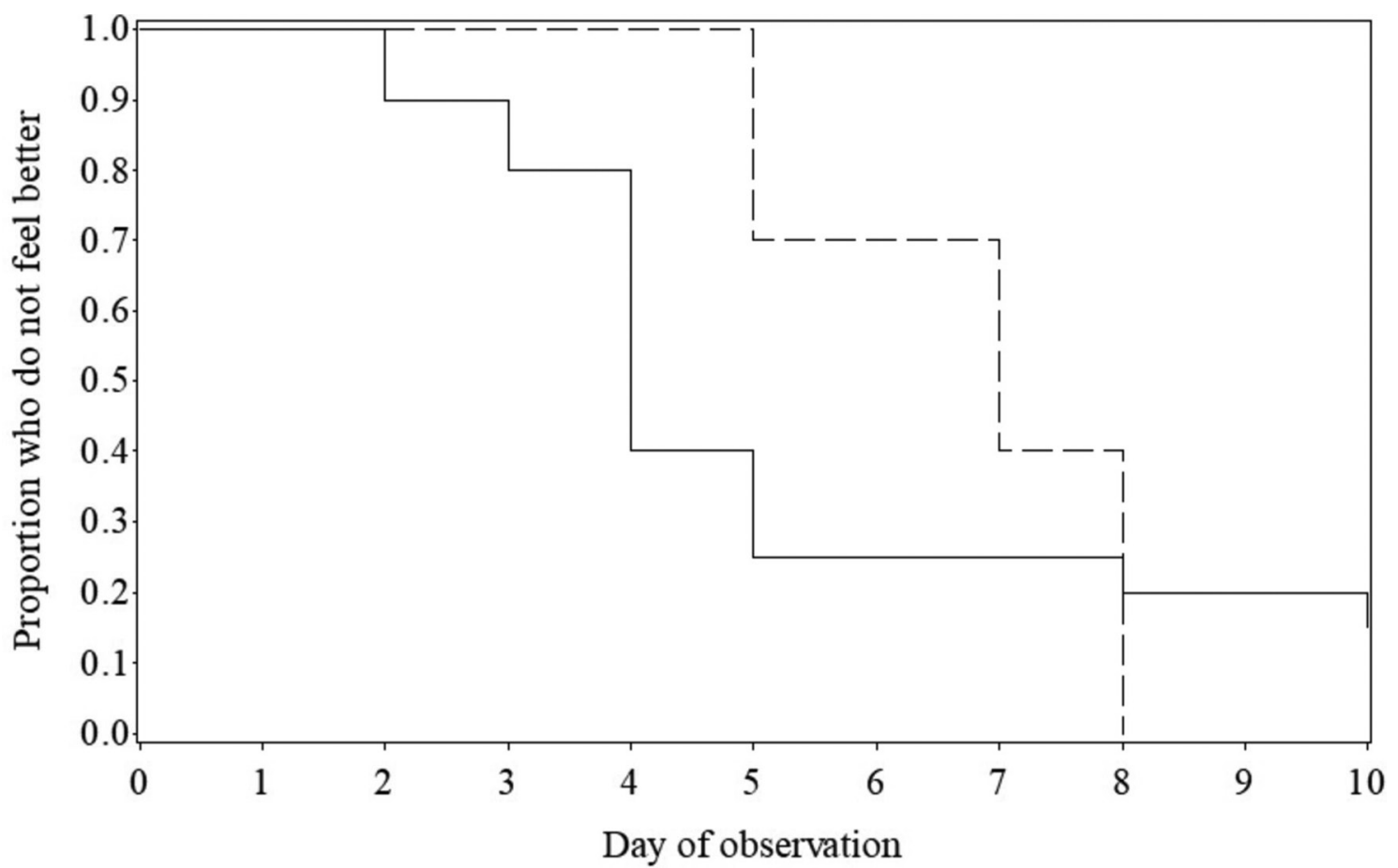

Figure 4 Kaplan-Meier survival curve for time to feeling better. Solid line represents rhinothermy group, while dotted line represents control.

and weight of the nasal interface. It also scored favourably with respect to ease of application but was found to be moderately noisy. Overall, participants scored a mean (SD) of 32.4 (27.4) units on a scale of likely future use where 0 was very likely and 100 was very unlikely.

\section{DISCUSSION}

This study has shown that an RCT of rhinothermy via high flow nasal cannulae in the treatment of the common cold is feasible. The study fully recruited 30 participants within an 8-week period, and all completed the study. Adherence to rhinothermy treatment was good while participants were symptomatic.

\begin{tabular}{|c|c|c|c|}
\hline PCR & All $(n=30)$ & $\begin{array}{l}\text { Rhinothermy } \\
(\mathrm{n}=20)\end{array}$ & $\begin{array}{l}\text { Contro } \\
(n=10)\end{array}$ \\
\hline Positive & $16(53.3)$ & $11(55.0)$ & $5(50.0)$ \\
\hline $\begin{array}{l}\text { Human rhinovirus } \\
\text { (HRV) }\end{array}$ & $10(33.3)$ & $6(30.0)$ & $4(40.0)$ \\
\hline Coronavirus & $1(3.3)$ & $0(0.0)$ & $1(10.0)$ \\
\hline Parainfluenza virus & $2(6.7)$ & $1(5.0)$ & $1(10.0)$ \\
\hline $\begin{array}{l}\text { Respiratory syncytial } \\
\text { virus }\end{array}$ & $3(10)$ & $3(15.0)$ & $0(0.0)$ \\
\hline Metapneumovirus & $1(3.3)$ & $1(5.0)$ & $0(0.0)$ \\
\hline Negative & $14(46.7)$ & $9(45.0)$ & $5(50.0)$ \\
\hline
\end{tabular}

All values given as $\mathrm{n}(\%)$.
This is the first study to investigate rhinothermy using the modified myAIRVO 2 device, and strengths include the randomised controlled design and the complete follow-up of all participants. Participants in this study were recruited primarily through social media so the results should be generalisable to people in the community with symptoms of the common cold.

Three main limitations apply to these results. First, this study was designed as a feasibility study that means that although the modified Jackson score was a prespecified

\begin{tabular}{|c|c|c|c|}
\hline Question & Mean (SD) & Median (IQR) & $\begin{array}{l}\text { Min to } \\
\max \end{array}$ \\
\hline $\begin{array}{l}\text { Ease of connecting } \\
\text { components }\end{array}$ & $16.8(21.6)$ & $5.5(2.0-22.0)$ & 0 to 67 \\
\hline Ease of cleaning* & $16.9(17.6)$ & $9.5(3.0-41.0)$ & 0 to 48 \\
\hline Overall comfort & $41.3(23.7)$ & $34.5(27.5-64.0)$ & 0 to 79 \\
\hline $\begin{array}{l}\text { Moisture in nasal } \\
\text { passages }\end{array}$ & $37.1(27.2)$ & $29.5(14.5-55.5)$ & 0 to 92 \\
\hline $\begin{array}{l}\text { Weight of nasal } \\
\text { cannula }\end{array}$ & $29.6(24.0)$ & $26.0(6.0-49.0)$ & 0 to 72 \\
\hline Noisiness & $54.0(26.4)$ & $57.5(35.5-72.0)$ & 0 to 95 \\
\hline Ease of application & $14.4(16.9)$ & $8.0(2.0-20.0)$ & 0 to 59 \\
\hline $\begin{array}{l}\text { Likelihood of reusing } \\
\text { rhinothermy }\end{array}$ & $32.4(27.4)$ & 26.5 (11.0-49.5) & 0 to 86 \\
\hline
\end{tabular}

All questions were scored from 0 to 100 with low scores representing greater acceptability. $n=20$ unless otherwise stated.

${ }^{*} \mathrm{n}=18$. 
efficacy outcome measure, we did not formally specify it as the primary outcome measure. Second, due to the nature of the intervention, we were unable to blind participants and investigators to the allocated intervention and a future study aiming to replicate these results should explore the option of sham rhinothermy. Finally, the small sample size means that although this study found benefits for the new treatment, the results are relatively 'fragile', in that the modified Jackson score would only need to change in a small number of participants who received the rhinothermy intervention to match the control group for our conclusions to be different. ${ }^{33} 34 \mathrm{~A}$ formal fragility index cannot be calculated for the change in modified Jackson score as it is not a dichotomous variable. The complete follow-up of all participants helps protect against this fragility; however, due to the above limitations a larger replication study is required.

The development of the myAIRVO 2 device with the capacity to deliver $100 \%$ humidified air at $41^{\circ} \mathrm{C}$ at high flow direct to the upper airways through wide bore nasal cannulae presented the opportunity to investigate the efficacy of rhinothermy again, after a lack of RCTs for over 20 years. The pragmatic regimen used in this study was based on available in vitro and in vivo studies. A temperature of $41^{\circ} \mathrm{C}$ was used as although there is a dose dependent reduction in $\mathrm{HRV}$ replication up to temperatures of $43^{\circ} \mathrm{C}-45^{\circ} \mathrm{C},{ }^{1516}$ temperatures of around $43^{\circ} \mathrm{C}$ are prone to cause minor mucosal burns. ${ }^{16}$ Two-hour sessions were used due to the evidence that the shortest duration of inhibition of HRV replication is observed with $30 \mathrm{~min}$ rather than $60 \mathrm{~min}$ of hyperthermia in vitro. ${ }^{16}$ Repeat sessions were administered over 5 days, as in vitro the effect of hyperthermia is transient, with HRV yields from hyperthermic treated cells reaching baseline levels within 24-48 hours. ${ }^{15}$

This rhinothermy regimen was well tolerated. Participants reported it was comfortable and easy to use and apply, although somewhat noisy. These are similar to observations made in our earlier tolerability of the myAIRVO device in healthy volunteers. ${ }^{26}$ In the current study, participants used the myAIRVO 2 device for 2 hours per day on average throughout the 5-day treatment period and for 2.6 hours on the days of use. As instructed, not all participants used the device late in the treatment course once their symptoms had improved. Around a third of participants reduced the flow rate to $25 \mathrm{~L} / \mathrm{min}$ or $30 \mathrm{~L} /$ min suggesting that giving participants the option of reducing the flow from the initial $35 \mathrm{~L} / \mathrm{min}$, or having a lower starting flow, is worthwhile.

Although not a primary outcome for the study, rhinothermy with the modified myAIRVO 2 device reduced common cold symptoms compared with low-dose vitamin C treatment.

The difference between the randomised groups was observed within 24 hours, so that the efficacy of single dose regimens or sessions over a period of 2-3 days, which may enhance its implementation in clinical practice, will be worth further study. However, while alternative rhinothermy regimens will need to be assessed, our study findings suggest that the regimen used in this feasibility study should also be used in future RCTs.

Our study has also been informative in terms of the likely number of participants required in an RCT. We have estimated the change in modified Jackson score that denotes substantial clinical benefit to be a 5-unit change. Based on the largest confidence limit for an SD of 6.6 (from the upper CI of the Jackson score at day 5), a sample size of 76 in each group allows the detection of a difference of 3.5 units, with $90 \%$ power and a type I error rate of $5 \%$. Allowing for a $10 \%$ dropout rate, a total of 85 participants would need to be randomised to each group. This would require a total of 170 participants in an RCT investigating two randomised treatments.

A specific respiratory virus was identified by PCR in $16 / 30(53 \%)$ of participants, with the majority being rhinovirus. This respiratory virus detection rate was comparable with previous studies that report detection rates between $43 \%$ and $64 \% .{ }^{35-37}$ We found no difference in the treatment effect between participants who were nasal swab PCR positive or negative, indicating that our findings are generalisable to symptomatic common colds and not restricted to those with positive virus detection. It also suggests that the efficacy may also relate to non-temperature-related antiviral effects, such as humidification of the airways and enhanced mucociliary clearance.

\section{CONCLUSION}

This study shows that an RCT of rhinothermy with the modified myAIRVO 2 device is feasible, that rhinothermy is well tolerated and that the estimated change denoting substantial clinical benefit for the modified Jackson score is a 5-unit change. Our observation that rhinothermy improved symptoms now requires replication in a larger study of common colds. Investigation of the efficacy of rhinothermy in the treatment of influenza is also a priority, in view of the temperature-sensitivity of influenza viruses $^{38-40}$ and the major public health burden of influenza worldwide. ${ }^{41} 42$

Contributors JF was the principal investigator for the study, is guarantor for the study and affirms that this manuscript is an honest, accurate and transparent account of the study being reported; that no important aspects of the study have been omitted; and that any discrepancies from the study as planned and registered have been explained. JF, SvdH, SM and GB were investigators on the study and collected the data. MW performed the statistical analysis. SvdH wrote the first draft of the manuscript. RB conceived the study and wrote the first draft of the protocol. All authors contributed to study design, interpretation of results, manuscript writing and reviewed the final manuscript prior to submission. All authors had full access to all of the data (including statistical reports and tables) in the study and can take responsibility for the integrity of the data and the accuracy of the data analysis. No writing assistance was received.

Funding The study was sponsored by Fisher \& Paykel Healthcare Limited. The MRINZ receives Health Research Council of New Zealand Independent Research Organisation funding.

Disclaimer The sponsor was given the opportunity to comment on the manuscript prior to submission but had no role in the collection, analysis and interpretation of data; in the writing of the report; or in the decision to submit the article for publication. 
Competing interests None declared.

Patient consent Not required.

Ethics approval The study was approved by the Northern B Ethics Health and Disability Ethics Committee of New Zealand (16/NTB/47).

Provenance and peer review Not commissioned; externally peer reviewed.

Data sharing statement Relevant anonymised patient level data are available on reasonable request.

Open Access This is an Open Access article distributed in accordance with the Creative Commons Attribution Non Commercial (CC BY-NC 4.0) license, which permits others to distribute, remix, adapt, build upon this work non-commercially, and license their derivative works on different terms, provided the original work is properly cited and the use is non-commercial. See: http://creativecommons.org/ licenses/by-nc/4.0/

(C) Article author(s) (or their employer(s) unless otherwise stated in the text of the article) 2018. All rights reserved. No commercial use is permitted unless otherwise expressly granted.

\section{REFERENCES}

1. Heikkinen T, Järvinen A. The common cold. Lancet 2003;361:51-9.

2. Lessler J, Reich NG, Brookmeyer R, et al. Incubation periods of acute respiratory viral infections: a systematic review. Lancet Infect Dis 2009;9:291-300.

3. Jacobs SE, Lamson DM, St George K, Kirsten S, et al. Human rhinoviruses. Clin Microbiol Rev 2013;26:135-62.

4. Fendrick AM, Monto AS, Nightengale B, et al. The economic burden of non-influenza-related viral respiratory tract infection in the United States. Arch Intern Med 2003;163:487-94

5. Bramley TJ, Lerner D, Sames M. Productivity losses related to the common cold. J Occup Environ Med 2002;44:822-9.

6. Arruda E, Pitkäranta A, Witek TJ, et al. Frequency and natural history of rhinovirus infections in adults during autumn. J Clin Microbiol 1997;35:2864-8.

7. Corne JM, Marshall C, Smith S, et al. Frequency, severity, and duration of rhinovirus infections in asthmatic and non-asthmatic individuals: a longitudinal cohort study. Lancet 2002;359:831-4.

8. Seemungal T, Harper-Owen R, Bhowmik A, et al. Respiratory viruses, symptoms, and inflammatory markers in acute exacerbations and stable chronic obstructive pulmonary disease. Am J Respir Crit Care Med 2001;164:1618-23.

9. Karsch-Völk M, Barrett B, Kiefer D, et al. Echinacea for preventing and treating the common cold. Cochrane Database Syst Rev 2014:CD000530.

10. De Sutter Al, Saraswat A, van Driel ML. Antihistamines for the common cold. Cochrane Database Syst Rev 2015:CD009345.

11. Hayward G, Thompson MJ, Perera R, et al. Corticosteroids for the common cold. Cochrane Database Syst Rev 2015;10:1-26.

12. Kenealy T, Arroll B, Kenealy T. Antibiotics for the common cold and acute purulent rhinitis. Cochrane Database Syst Rev 2013:CD000247.

13. Hemilä $\mathrm{H}$, Chalker E. Vitamin $\mathrm{C}$ for preventing and treating the common cold. Cochrane Database Syst Rev 2013:CD000980.

14. Rohde GG. Rhinovirus vaccination: the case in favour. Eur Respir J 2011;37:3-4.

15. Conti C, De Marco A, Mastromarino P, et al. Antiviral effect of hyperthermic treatment in rhinovirus infection. Antimicrob Agents Chemother 1999;43:822-9.

16. Forstall GJ, Macknin ML, Yen-Lieberman BR, et al. Effect of inhaling heated vapor on symptoms of the common cold. JAMA 1994;271:1109-11.

17. Schroth MK, Grimm E, Frindt P, et al. Rhinovirus replication causes RANTES production in primary bronchial epithelial cells. Am J Respir Cell Mol Biol 1999;20:1220-8.

18. Stott EJ, Heath GF. Factors affecting the growth of Rhinovirus 2 in suspension cultures of L132 cells. J Gen Virol 1970;6:15-24.
19. Foxman EF, Storer JA, Fitzgerald ME, et al. Temperature-dependent innate defense against the common cold virus limits viral replication at warm temperature in mouse airway cells. Proc Natl Acad Sci U S A 2015;112:827-32.

20. Heron I, Berg K. The actions of interferon are potentiated at elevated temperature. Nature 1978;274:508-10.

21. Singh M. Heated, humidified air for the common cold. Cochrane Database Syst Rev 2013:CD001728.

22. Tyrrell D, Barrow I, Arthur J. Local hyperthermia benefits natural and experimental common colds. BMJ 1989;298:1280-3.

23. Ophir D, Elad Y. Effects of steam inhalation on nasal patency and nasal symptoms in patients with the common cold. Am J Otolaryngol 1987:8:149-53

24. Hendley JO, Abbott RD, Beasley PP, et al. Effect of inhalation of hot humidified air on experimental rhinovirus infection. JAMA 1994;271:1112-3.

25. Macknin ML, Mathew S, Medendorp SV. Effect of inhaling heated vapor on symptoms of the common cold. JAMA 1990;264:989-91.

26. Bibby S, Reddy S, Cripps T, et al. Tolerability of nasal delivery of humidified and warmed air at different temperatures: a randomised double-blind pilot study. Pulm Med 2016;2016:1-7.

27. Jackson GG, Dowling HF, Spiesman IG, et al. Transmission of the common cold to volunteers under controlled conditions. I. The common cold as a clinical entity. AMA Arch Intern Med 1958;101:267-78.

28. Farr BM, Gwaltney JM, Hendley JO, et al. A randomized controlled trial of glucocorticoid prophylaxis against experimental rhinovirus infection. J Infect Dis 1990;162:1173-7.

29. Gwaltney JM, Hendley JO, Patrie JT. Symptom severity patterns in experimental common colds and their usefulness in timing onset of illness in natural colds. Clin Infect Dis 2003;36:714-23.

30. Harris PA, Taylor R, Thielke R, et al. Research electronic data capture (REDCap)--a metadata-driven methodology and workflow process for providing translational research informatics support. J Biomed Inform 2009;42:377-81.

31. Zannikos S, Lee L, Smith HE. Minimum clinically important difference and substantial clinical benefit: does one size fit all diagnoses and patients? Semin Spine Surg 2014;26:8-11.

32. Carreon LY, Glassman SD, Campbell MJ, et al. Neck Disability Index short form-36 physical component summary, and pain scales for neck and arm pain: the minimum clinically important difference and substantial clinical benefit after cervical spine fusion. Spine $J$ 2010;10:469-74

33. Walsh M, Srinathan SK, McAuley DF, et al. The statistical significance of randomized controlled trial results is frequently fragile: a case for a fragility index. J Clin Epidemiol 2014;67:622-8.

34. Ridgeon EE, Young PJ, Bellomo R, et al. The fragility index in multicenter randomized controlled critical care trials. Crit Care Med 2016;44:1278-84.

35. Spyridaki IS, Christodoulou I, de Beer L, De BL, et al. Comparison of four nasal sampling methods for the detection of viral pathogens by RT-PCR-A GA(2)LEN project. J Virol Methods 2009;156:102-6.

36. Brittain-Long R, Westin J, Olofsson S, et al. Prospective evaluation of a novel multiplex real-time PCR assay for detection of fifteen respiratory pathogens-duration of symptoms significantly affects detection rate. J Clin Virol 2010;47:263-7.

37. Leekha S, Irish CL, Schneider SK, et al. Viral detection using a multiplex polymerase chain reaction-based assay in outpatients with upper respiratory infection. Diagn Microbiol Infect Dis 2013;75:169-73.

38. Chu CM, Tian SF, Ren GF, et al. Occurrence of temperature-sensitive influenza A viruses in nature. J Virol 1982;41:353-9.

39. Oxford JS, Corcoran T, Schild GC. Naturally occurring temperaturesensitive influenza $A$ viruses of the $\mathrm{H} 1 \mathrm{~N} 1$ and $\mathrm{H} 3 \mathrm{~N} 2$ subtypes. J Gen Virol 1980;48:383-9.

40. Kung HC, Jen KF, Yuan WC, et al. Influenza in China in 1977: recurrence of influenzavirus A subtype H1N1. Bull World Health Organ 1978;56:913-8.

41. World Health Organisation (WHO). Influenza (seasonal). WHO media centre. Fact Sheet No 211

42. Cox NJ, Subbarao K. Influenza. Lancet 1999;354:1277-82. 